Trivent Publishing

(C) The Authors, 2018

Available online at http:/ / trivent-publishing.eu/

TRIVENT

Series: Applied Ethics: From Bioethics to Environmental Ethics

\title{
Haematological Patients' Perception of Their Quality of Life
}

\author{
Alciona Sasu, ${ }^{1}$ Mircea Onel,,${ }^{2}$ Cristina Ghib-Para,${ }^{3}$ Ligia Piroș, 4 , Alin \\ Greluş, ${ }^{5}$ Coralia Cotoraci ${ }^{6}$ \\ ${ }^{1}$ Bioethics and Medical Deontology Department, \\ "Vasile Goldiş" Western University of Arad, Romania, alcionasasu@gmail.com \\ 2 Bioethics and Medical Deontology Department, \\ "Vasile Goldiş” Western University of Arad, Romania, mirceaonel@yahoo.com \\ ${ }^{3}$ Bioethics and Medical Deontology Department, \\ "Vasile Goldiş" Western University of Arad, Romania, paracris17@yahoo.com \\ ${ }^{4}$ Psychiatry Department, "Vasile Goldiș" Western University \\ of Arad, Romania, diana_ligia2000@yahoo.com \\ 5 Urology Department, "Vasile Goldiş" Western University \\ of Arad, Romania, alin_gvg@yahoo.com \\ ${ }^{6}$ Bioethics and Medical Deontology Department, \\ "Vasile Goldiş" Western University of Arad, Romania, ccotoraci@yahoo.com
}

Abstract: The therapeutic management of haematological patients dramatically changes the lives of patients. Quality of life is a modern concept which tries to belp the patient and refocuses doctors on improving not only the patient's symptoms and disease itself, but also the patient's overall well-being. This chapter will highlight the most important factors which can modify the onco-haematological patient's quality of life, stressing the importance of biological, psychological, and social factors. In identifying these factors, we used two standard questionnaires which evaluate the quality of life on various fields. The results showed that physical status, mental bealth, and social factors influence patient quality of life.

Keywords: quality of life; haematological patients; chemotherapy; oncologic diagnosis; psychotherapy.

This is an Open Access article distributed in accordance with the Creative Commons Attribution Non Commercial (CC-BY-NC-ND 4.0) license, which permits others to copy or share the article, provided original work is properly cited and that this is not done for commercial purposes. Users may not remix, transform, or build upon the material and may not distribute the modified material (bttp:/ / creativecommons.org/licenses/by-nc/4.0/) 


\title{
Haematological Patients' Perception of Their Quality of Life
}

\author{
Alciona Sasu, Mircea Onel, Cristina Ghib-Para, \\ Ligia Piroş, Alin Greluş, Coralia Cotoraci
}

\section{Introduction}

Haematological patients are usually complex patients, whether they have benign or malignant pathologies. The patients admitted in a Hematology Clinic are mostly oncohaematological patients, with severely altered health status, with multiple and frequent complications. Cancer diagnosis brings profound changes in the lives of the patient and their family. Cancer is a chronic illness that has a major impact on general activities, including social integration during and after treatment. Moreover, chemotherapy changes the general status of the patient and frequently involves patient admission into the hospital. During chemotherapy, the patient might have adverse reactions at an incidence depending on the drug administered and the patient's clinical background [1]. Chemotherapy toxicity can take several aspects, from asthenia to severe haematological, digestive, immunological, or dermatological complications. Moreover, toxicity can be early or tardive, thus influencing the patient's status immediately after (but also months after) treatment [2]. Life-changing diagnosis, modified life style, acceptance of diagnosis, psychological disturbances, prolonged hospitalisation, privation of family life and social life -these are only some of the challenges that oncological patients face shortly after diagnosis.

In the last decades, medicine has underlined the importance of quality of life [3], besides the specific indicators which stress patient outcome (e.g. survival rate, disease free survival, etc).

The concept of quality of life is hard to define and should be used with great discernment [4]. According to the World Health Organization, quality of life is given by the individuals' perceptions of their own social situations in the context of the cultural value systems they live in, as well as their own needs, standards, and aspirations [5]. Health related quality of life means physical, mental, social [6] and, more recently, spiritual well-being, as well as the ability of patients to perform their ordinary tasks in their everyday life [7].

Life quality assessment is done using varied fields with multiple indicators. 
Different models of quality of life assessment have been designed: some specific to certain pathologies [8], others trying to make a difference between function and health, assessing health in the context of permanent functional limitations [9].

Having all these data, haematologists and oncologists conduct a complex therapy management of their patients, combining the standard ethiologic therapy with the psychological, social, spiritual, or alternative therapies [10].

\section{Material and methods}

\section{Patients}

We conducted a prospective study over a period of 12 months (2015/2016) within a group of 50 patients admitted in the Department of Haematology of the Clinical County Emergency Hospital of Arad (Romania). They were patients diagnosed with oncohaematological diseases. The patients were divided into 3 lots:

Lot A - patients who underwent oncological treatment ( $\mathrm{n}=27)$;

Lot B - patients who underwent oncological and psychiatric treatment $(\mathrm{n}=$ $15)$;

Lot $\mathrm{C}$ - patients who underwent oncological and psychological treatment $(\mathrm{n}=8)$.

\section{Hypothesis}

In order to achieve the goal, we tested the following hypothesis:

Hypothesis 1 - physical health affects mental health status, social behaviour, and relationships;

Hypothesis 2 - mental health influences social activity;

Hypothesis 3 - oncological, psychiatric, and psychological treatment influences the state of mental health, general health, and quality of life of oncohaematological patients;

Hypothesis 4 - the emergence of a stress factor before the onset of illness affects mental health.

\section{Objective}

The main objective: Assessing the quality of life in oncohaematological patients and highlighting the emotional, physical, and social factors impacting on the indices that characterize the quality of life of these patients.

\section{Working documents}

1. Informed consent; 


\section{Alciona Sasu et al.}

Haematological Patients' Perception of Their Quality of Life

2. Anamnestic test;

3. Special psychological tests for quality of life assessment: MOS-SF-36 Medical Outcome Study-Short Form 36 (principal author John Ware Jr., 1992, having 8 fields: Physical functionality scale, Scale of problems caused by physical disorders, The social functionality scale, Scale of body pain, The mental health scale, Scale of problems caused by emotional states, Vitality scale, The general health scale) and SCL-90-R Symptom Checklist Revised (Derogatis, 1983, with 9 items: Somatization, Obsessive-compulsive disorder, Anxiety, Depression, Interpersonal sensitivity or sensitivity, Hostility, Phobic anxiety or phobias, Psihoticismus, Paranoid ideation). ${ }^{1}$

\section{Results}

The study included 50 subjects aged between 42 and 80 , with an average age of 65.08 years; $50 \%$ were women and $50 \%$ were men; $44 \%$ from urban areas and the remaining $56 \%$ from rural areas; $58 \%$ had elementary studies, $8 \%$ of subjects were without education, $16 \%$ had secondary education, and $18 \%$ had higher education. Concerning the income, $32 \%$ had a lower income than the mean economy income, $56 \%$ had a minimum per economy income, and $12 \%$ had an average per economy income.

The majority of $84 \%$ lived with their family, $16 \%$ lived alone, and no subject was institutionalized.

Of all participants, $32 \%$ had a history of psychiatric disease and the remaining $68 \%$ had no such history. Moreover, $4 \%$ of the subjects had a history of psychological trauma and the remaining $96 \%$ had no previous psychological trauma.

Of the study subjects, $6 \%$ often drank alcohol, another $16 \%$ occasionally drank alcohol, and the vast majority of $78 \%$ did not drink alcohol.

The group structure based on behaviour resulted from the question "What is your activity during the day?". The responders answered as following: 7.6\% preferred social withdrawal, $52.8 \%$ had minimum social activity and 39.6\% were socially active.

Concerning the therapy used for the patients, 54\% only had oncological treatment, $30 \%$ had oncological treatment associated with psychiatric treatment, and $16 \%$ had oncological treatment associated with psychological therapy (Fig 1).

${ }^{1}$ Authors used authorized sources for all these tests. 
Haematological Patients' Perception of their Quality of Life

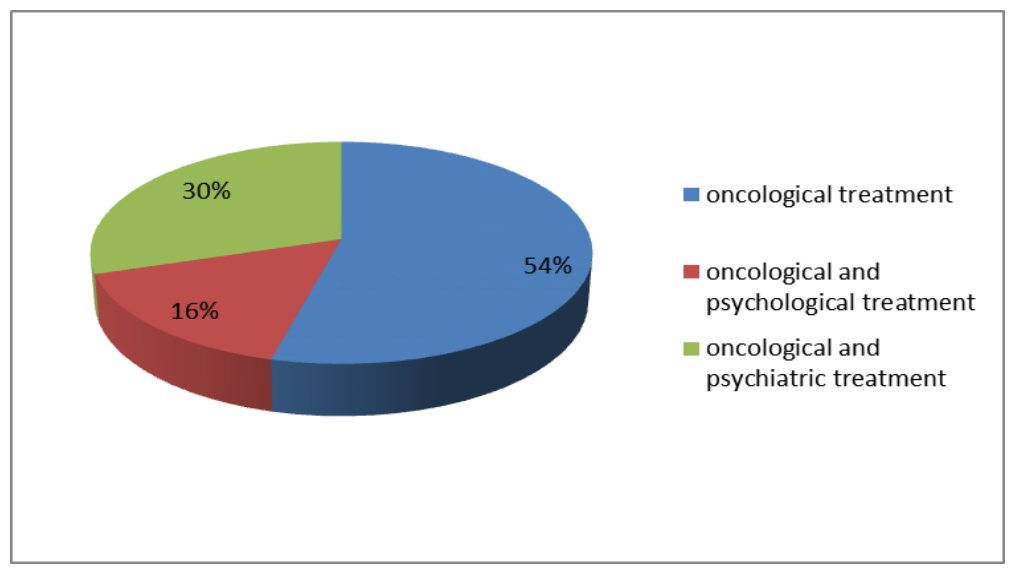

Fig. 1. The group structure according to the treatment

Descriptive analysis of the test and anamnestic results on the 4 plans

- Physical-biological plan

Somatization: reflects the distress which occurs as a result of the perception of bodily dysfunction, the symptoms being centred on the gastrointestinal, respiratory, muscular discomfort (Table 1). The higher the scores, the worse the quality of the person's life.

Table 1. Somatization according to SCL 90

\begin{tabular}{|l|c|}
\hline $\mathbf{N}$ & 50 \\
\hline Mean & 26.4800 \\
\hline Std. Deviation & 6.97880 \\
\hline Minimum & 14.00 \\
\hline Maximum & 44.00 \\
\hline
\end{tabular}

- The general health scale

Test SF 36: Score 5-25, the higher the scores, the better the quality of life (Table 2). 
Table 2. General Health Scale according to Questions 1, 2 and 11 (5- 25)

\begin{tabular}{|l|c|}
\hline N & 50 \\
\hline Mean & 15.1600 \\
\hline Std. Deviation & 2.78010 \\
\hline Minimum & 9.00 \\
\hline Maximum & 21.00 \\
\hline
\end{tabular}

In order to determine the general health level so that we can divide the participants into two groups, we calculated a general health score based on the answers to questions 1, 2 and 11 of SF. 36.

Subjects with a lower score than the median per group were included in the group of those with a lower health status, and those with a higher than median score were included in the group of those with a better health status.

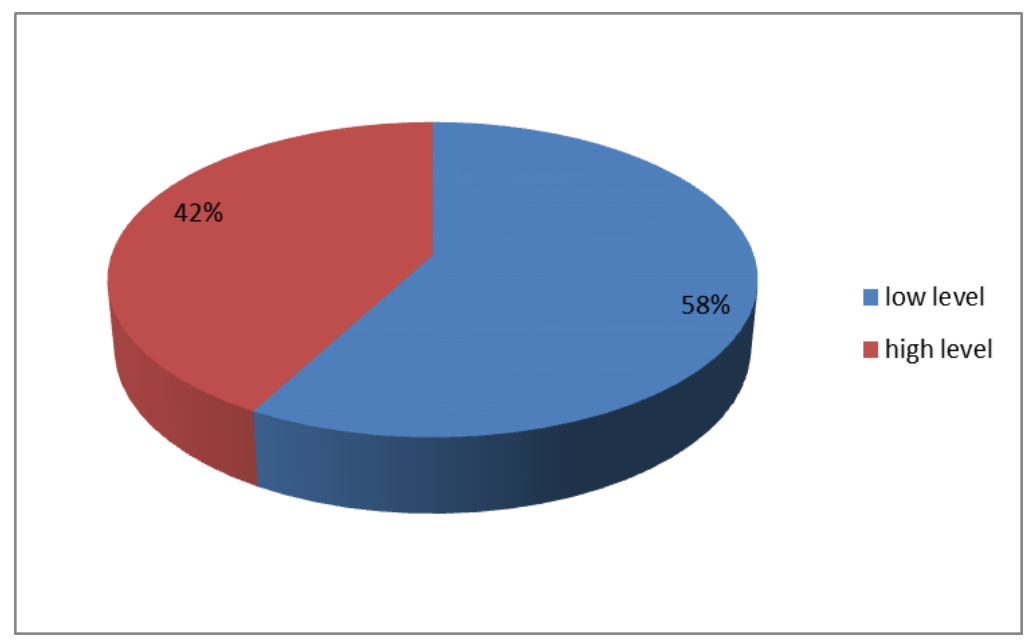

Fig. 2. Group structure according to the general health status

- Physical functionality scale: was described from question 3 SF 36, scores 10-30 (Table 3). The higher the scores, the better the quality of life. 
Haematological Patients' Perception of their Quality of Life

Table 3. Physical functionality scale according to SF 36

\begin{tabular}{|l|c|}
\hline $\mathrm{N}$ & 50 \\
\hline Mean & 17.1600 \\
\hline Std. Deviation & 5.28903 \\
\hline Minimum & 10.00 \\
\hline Maximum & 29.00 \\
\hline
\end{tabular}

- The social functionality scale: was described from question no. 6 of the SF 36 test (Fig 3): "In the last 4 weeks, to what extent have your physical health or emotional problems affected your ordinary activities related to family, friends, neighbours, or other groups of people?"

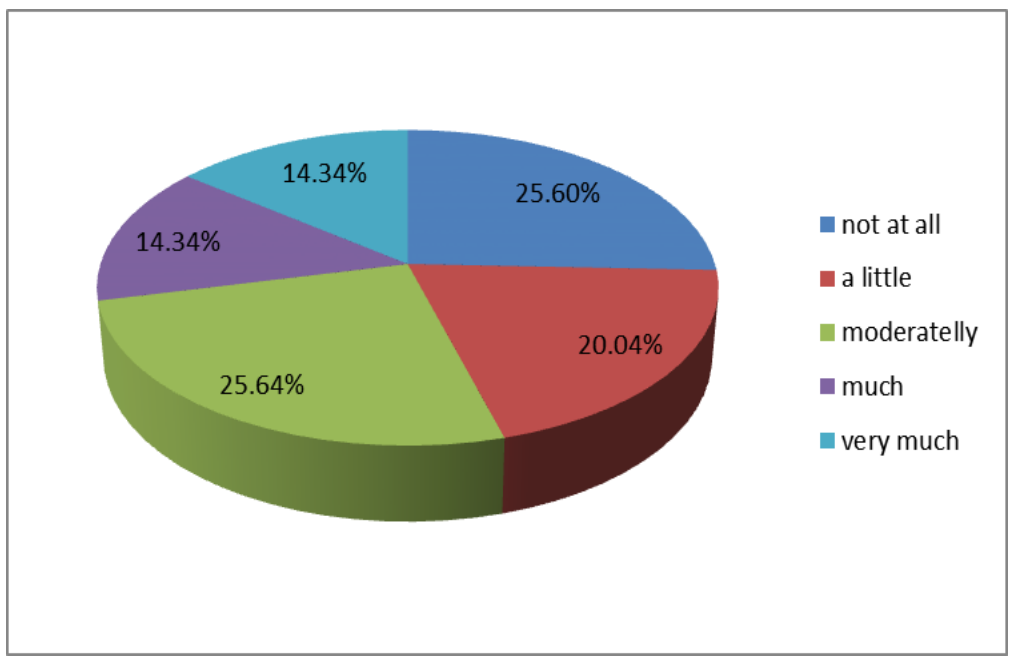

Fig. 3. Group structure according to social functionality scale

- Psycho-emotional plan

The results showed that $60 \%$ of the subjects had a happy childhood and $40 \%$ had an unhappy childhood.

Concerning the existence of an unfortunate event (stressor) before the onset of the disease, the results showed that $66 \%$ of the subjects experienced such an event and 34\% have not.

For the existence of obsessive-compulsive disorder, anxiety, depression, sensitivity, interpersonal hostility, phobia, psychoticism, paranoid ideation ( $\mathrm{Scl}$ 90) the results are presented in Table 4 . The higher the scores, the worse the quality of life. 
Table 4. Group structure according to psychiatric problems

\begin{tabular}{|c|c|c|c|c|c|c|c|c|}
\hline & 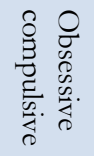 & $\begin{array}{l}\nabla \\
0 \\
0 \\
0 \\
0 \\
0 \\
0 \\
0\end{array}$ & 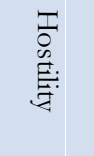 & 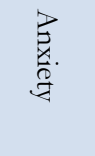 & 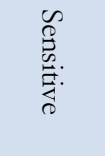 & $\frac{\widetilde{\sigma}}{\frac{\sigma}{0}}$ & 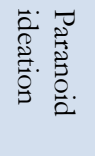 & 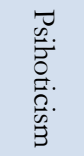 \\
\hline $\mathrm{N}$ & 15 & 15 & 15 & 15 & 15 & 15 & 15 & 15 \\
\hline Mean & $\begin{array}{c}16.133 \\
33\end{array}$ & $\begin{array}{c}19.333 \\
3\end{array}$ & 8.6000 & 8.6000 & 13.0000 & $\begin{array}{c}10.266 \\
7\end{array}$ & 8.6667 & $\begin{array}{c}12.333 \\
3\end{array}$ \\
\hline $\begin{array}{l}\text { Std. } \\
\text { Devia } \\
\text {-tion }\end{array}$ & $\begin{array}{c}6.0694 \\
4\end{array}$ & $\begin{array}{c}8.8855 \\
2\end{array}$ & $\begin{array}{c}3.0659 \\
4\end{array}$ & $\begin{array}{c}6.6811 \\
7\end{array}$ & 5.51621 & $\begin{array}{c}5.5093 \\
0\end{array}$ & $\begin{array}{c}3.3735 \\
7\end{array}$ & $\begin{array}{c}4.1518 \\
8\end{array}$ \\
\hline Min. & 10.00 & 12.00 & 6.00 & 10.00 & 9.00 & 7.00 & 6.00 & 10.00 \\
\hline Max. & 26.00 & 34.00 & 14.00 & 31.00 & 25.00 & 27.00 & 15.00 & 23.00 \\
\hline
\end{tabular}

- The level of mental health

In order to determine the mental health level so that we can divide the participants into two groups, we calculated a mental health score based on the score of the Scl 90 test. Subjects with a lower score than the median per group were included in the group of those with a higher level of health and those with a higher than median score were included in the group of those with a lower health level. The results showed that $50 \%$ had a low level of mental health and $50 \%$ a had high level of mental health.

Comparative analysis of the testological and anamnestic results

- Impact of overall health on the social plan (Fig. 4) 
Haematological Patients' Perception of their Quality of Life

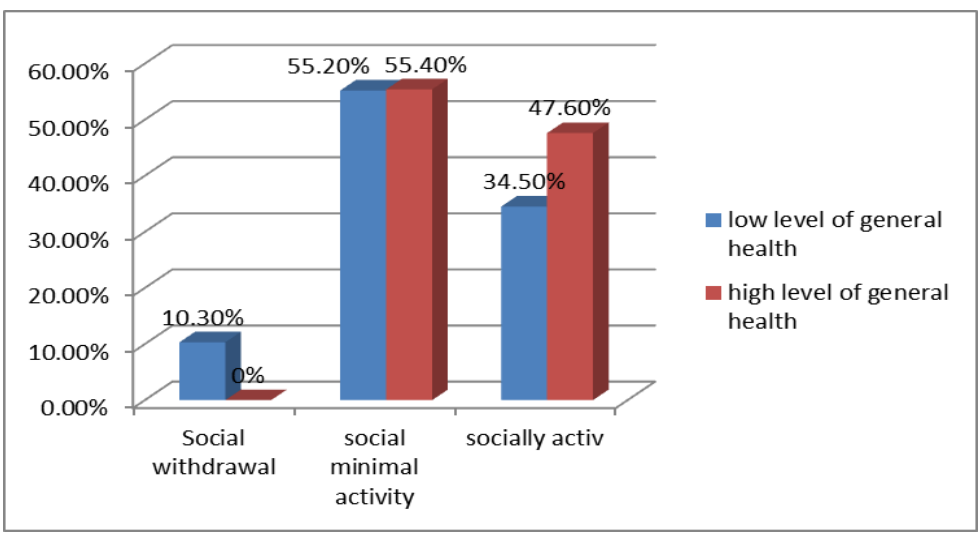

Fig. 4. The influence of physical-general health on behaviour

- The influence of the physical-general health state on the social function: was deduced from the question: "In the last 4 weeks, did you have moments when your physical or emotional health has affected your social activities (e.g. visits to friends, relatives etc.)?” (Fig. 5).

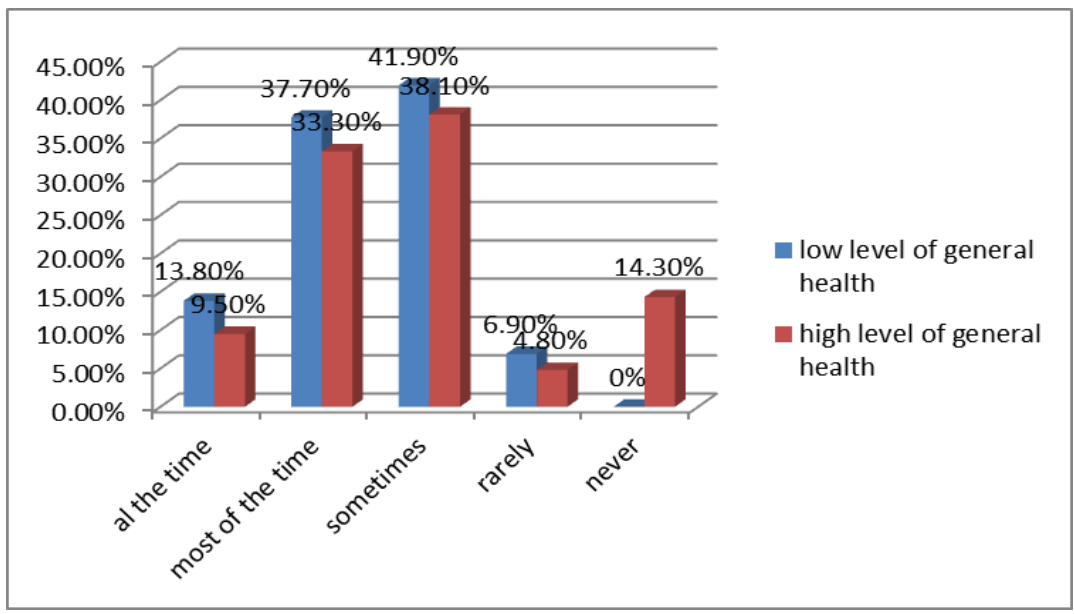

Fig. 5. The influence of physical-general health on social function

- The impact of mental health on the social plan (Fig. 6) 


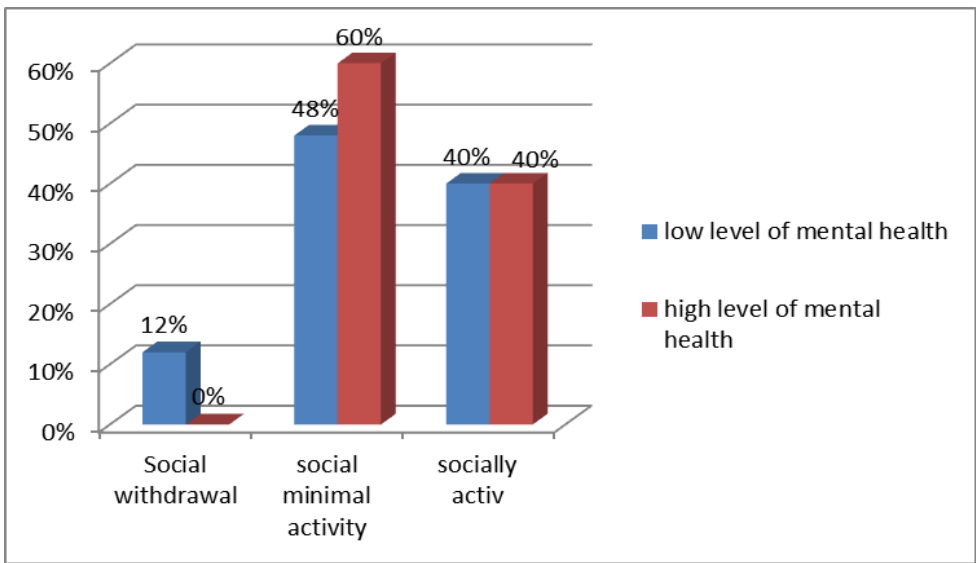

Fig. 6. The influence of mental health on behaviour

- The influence of mental health status on social function: was deduced from the question "In the past 4 weeks, did you have moments when your physical or emotional health has affected your social activities (e.g. visits to friends, relatives, etc.)?” (Fig. 7).

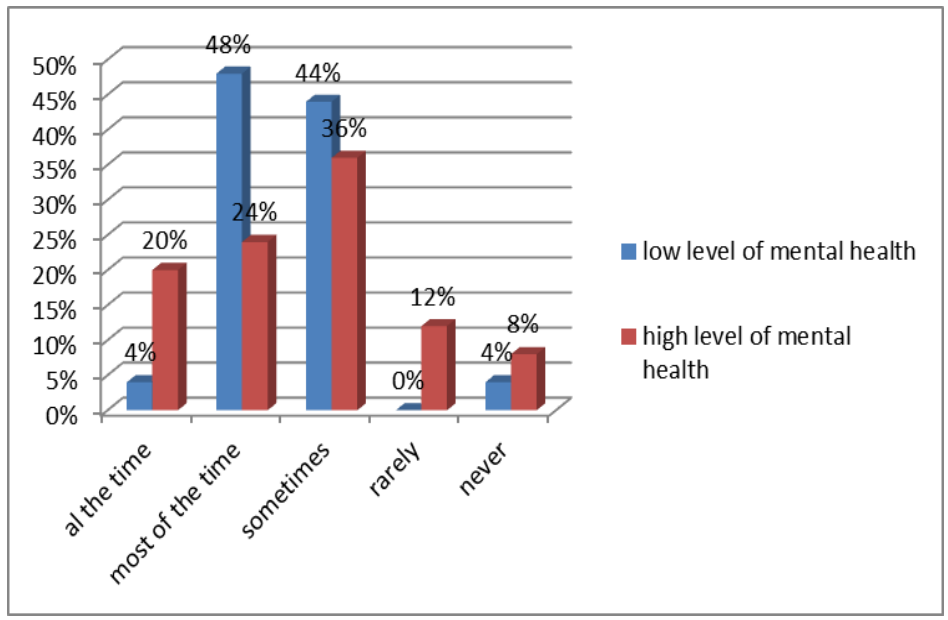

Fig. 7. Influence of mental health status on social function

- The influence of treatment on mental health (Fig. 8) 
Haematological Patients' Perception of their Quality of Life

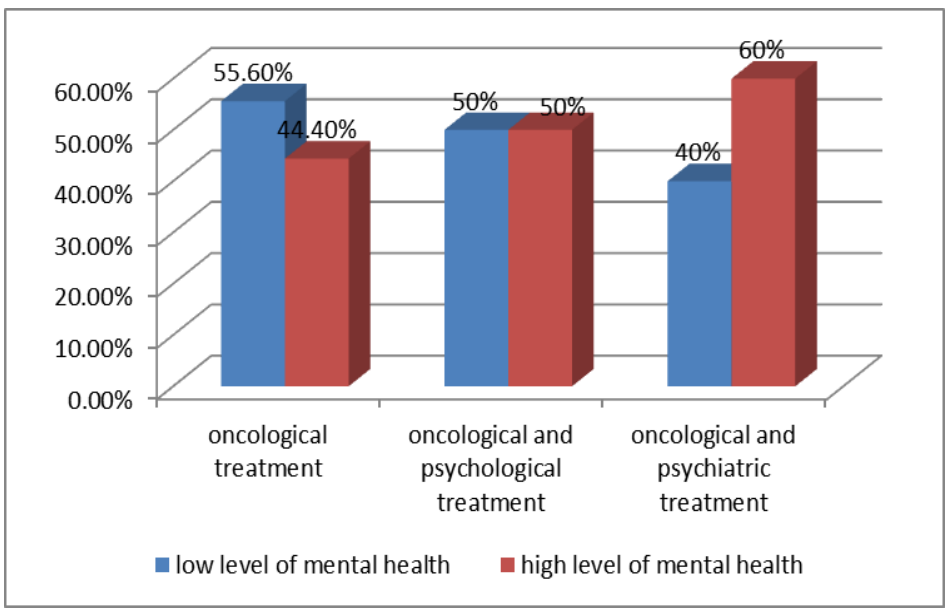

Fig 8. The influence of treatment on mental health

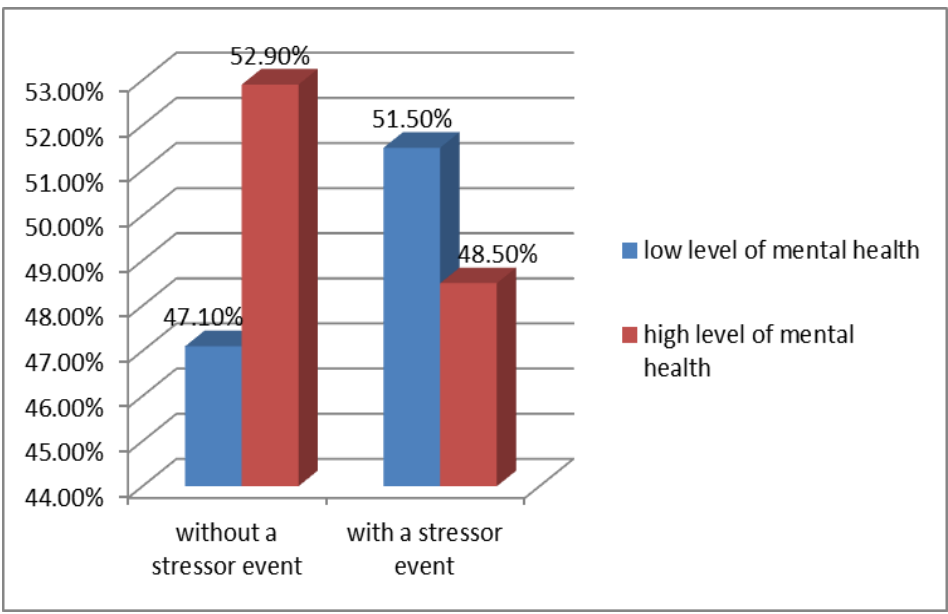

Fig. 9. The influence of the presence of an unfortunate (stressor) event before the onset of illness, on mental health 


\section{Discussion}

Quality of life is a topic which has become increasingly important in recent years, demonstrating the need to extend clinical research and practice beyond simply assessing the size of symptoms. Furthermore, it has shown that the goal of treatment is not only reducing symptoms but also improving the quality of life [11].

Among the most important factors influencing the quality of life in the subjects of this study were the biological factors in which we can intervene by treatment (depression, anxiety, somatic pathology, oncological pathology). Psychiatric comorbidities were present in $32 \%$ of male and female subjects. $4 \%$ of the subjects suffered physical trauma in their past, which is a factor that reduces quality of life. Biological factors (somatic and psychiatric) can be successfully intervened upon, thus improving the patients' quality of life.

As stated before, of the study subjects, $54 \%$ had only oncological treatment, 30\% had oncological treatment with psychiatric treatment, and the remaining 16\% had oncological treatment with psychological treatment (Fig 1). Recent studies support the hypothesis that when combined with chemotherapy, psychiatric therapy can improve the effects of chemotherapeutic agents, suggesting that SSRI (selective serotonin reuptake inhibitors) antidepressants have the potential to chemosensitize cancer cells in chemotherapy regimens [12-13].

\section{Psychological factors}

The psychological state of cancer patients is correlated with the stages of the disease: there is a decrease in depression and anxiety rates when patients are in the remission phase and they increase in the relapses of the disease. The stigmatization of psychiatric and oncological pathology can lead to a demonstrated decrease in patient compliance, which falls in a vicious circle: each new recurrence accentuating the stigma of the entourage. Humour, optimism, resilience, memory of past positive experiences, positive suggestiveness, internal controlling site (assuming responsibility for monitoring the first symptoms of a new episode, adherence to drug treatment) are factors which can be cultivated through psychological education $(16 \%$ constantly participating in psychotherapy programs) in order to determine the most favourable development of the underlying disease and to improve the quality of life [14].

\section{Socio-economic factors}

Social support can be granted by family, colleagues, friends, but also by institutions such as church or various forms of social protection. Social 
support plays a protective role concerning stressors predisposing to depression and anxiety (Table 4) [15].

Among the study subjects, the majority of $84 \%$ live with family and the remaining $16 \%$ live alone. Of the participants in the study, $7.6 \%$ prefer loneliness, $52.8 \%$ have a minimum social activity and the rest of $39.6 \%$ are socially active. The subjects have proved to have good social support, the majority living with the family and contributing to the improvement of the quality of life.

Somatization reflects the distress which occurs as a result of the perception of bodily dysfunction, accusations being centred on gastrointestinal, respiratory, and muscular discomfort.

The scores on this group of subjects (Table 1) ranged from 14 to 44, averaging 26.48, concluding that subjects with psychiatric comorbidities have a very low perception of quality of life.

Of the study subjects, concerning the mental health, $50 \%$ had low mental health level and the rest high level of mental health. Quality of life is inversely proportional to the score obtained from the Scl 90 testology for subjects suffering from psychological disorders, with approximately half of the subjects achieving high scores following the test.

The test being applied during hospitalization, at the time of crisis of the subject, reveals that this period is a critical moment in which the declared quality of life is close to the minimum.

The scores obtained in the SF 36 physical scale on this group of subjects ranged from 10 to 29 with an average of 17.16 , which demonstrates that in general the oncohaematological patient's functionality is influenced, the quality of life being altered due to this aspect.

The scale of social functionality applied at the time of hospitalization has better quality of life scores, only one-third of subjects feel the disease has affected their relationship with those around them.

The data obtained helps conclude that childhood contributes to the mental health of the subject and, implicitly, to the quality of life of the subject.

It was observed that subjects who had a stressor before the onset of the disease $(66 \%)$ had a lower level of mental health than those who did not have such an event (34\%).

\section{Conclusions}

The data of this study shows that physical health influences the state of mental health, behaviour, and relations on the social plan. For all the mental health dimensions of the Scl 90 test, individuals with low physical health have been found to have scores higher than those with a high level of physical health, which means that people with reduced physical health are psychologically 


\section{Alciona Sasu et al.}

Haematological Patients' Perception of Their Quality of Life

affected more than those with a good physical health. Moreover, subjects with a high level of general physical health are more socially active than those with a low level of general physical health. It has been observed that subjects with a high level of general physical health have better relationships with people surrounding them than those with a low level of general physical health.

Mental health influences behaviour, social function, and relationships with people around. Subjects with a low mental health level are more sociallywithdrawn than those with an increased mental health level (Fig. 6, 7). Subjects with an increased mental health level have better relationships with people around them than those with a low mental health level.

Oncological, psychiatric, and psychological treatment, influences the state of mental health, general health, and quality of life of oncohaematological patients. Subjects who have undergone psychiatric and psychological treatment together with oncological treatment have a higher mental health level than those who have only had oncological treatment (Fig 8).

The emergence of a stressor factor before the onset of the disease, influences mental health. Subjects who had a stressor before the onset of the disease have a lower level of mental health than those who did not have such events before the onset of the disease.

It is clearly shown that physical status and emotional status influence the patient's quality of life. There are also other keys - such as the stressor event before the onset of the disease - that should evaluated more deeply. Moreover, the mental status of the patient resulted from his history can be relevant when appreciating the psychological influence of cancer on the patient's emotional status. The patient's family plays an important role in patient's social quality of life.

This study helps in the complex evaluation of an oncohaematological patient, highlighting the main emotional and social factors which affect the indices which characterize the quality of life of oncohaematological patients in order to develop strategies to prevent and improve it.

\section{References}

[1] Deepti Chopra, Harmeet S. Rehan, Vibha Sharma, and Ritu Mishra, "Chemotherapy-induced adverse drug reactions in oncology patients: A prospective observational survey", Indian J Med Paediatr Oncol. 2016 JanMar; 37(1): 42-46. doi: 10.4103/0971-5851.177015 PMCID: PMC4795375

[2] Ajitha Sharma, K. Meena Kumari,1 Hasitha Diana Manohar,1 K. L. Bairy,1 and Joseph Thomas, "Pattern of adverse drug reactions due to cancer chemotherapy in a tertiary care hospital in South India", Perspect 
Clin Res. 2015 Apr-Jun; 6(2): 109-115. doi: 10.4103/2229-3485.154014, PMCID: PMC4394577

[3] Pennacchini M, Bertolaso M, Elvira MM, De Marinis MG "A brief history of the Quality of Life: its use in medicine and in philosophy", Clin Ter. 2011;162(3):e99-e103

[4] Marcel W.M, "Definitions of Quality of Life: What Has Happened and How to Move On”, Top Spinal Cord Inj Rehabil. 2014 Summer; 20(3): 167180. Published online 2014 Aug 11. doi: 10.1310/sci2003-167, PMCID: PMC4257148

[5] WHOQOL Group. Development of the World Health Organization WHOQOL-BREF quality of life assessment. Psychol Med. 1998;28:551558. [PubMed]

[6] http://www.healthmeasures.net/explore-measurementsystems/promis/intro-to-promis

[7] Bottomley, Andrew, "The Cancer Patient and Quality of Life", The Oncologist, 7 (2): 120-125. ISSN 1083-

7159. PMID 11961195. doi:10.1634/theoncologist.7-2-120.

Retrieved 2015-05-03. (April 2002).

[8] Marcel W.M. Post, "Definitions of Quality of Life: What Has Happened and How to Move On", Top Spinal Cord Inj Rehabil. 2014 Summer; 20(3): 167-180. Published online 2014 Aug 11. doi: 10.1310/sci2003-167, PMCID: PMC4257148

[9] Krahn GL, Suzuki R, Horner-Johnson W, "Self-rated health in persons with spinal cord injury: relationship of secondary conditions, function and health status", Qual Life Res. 2009 Jun; 18(5):575-84

[10] Katarzyna Cieślak, "Professional psychological support and psychotherapy methods for oncology patients. Basic concepts and issues", Rep Pract Oncol Radiother. 2013 May; 18(3): 121-126. Published online 2013 May 3. doi: 10.1016/j.rpor.2012.08.002 PMCID: PMC3863260

[11] Krouse, H. J., "Efficacy of video education for patients and caregivers", ORL-Head \&Neck Nursing, no. 21(1), 2003.

[12] Scuster, C., Fernbach, N., Rix, U., Superti-Furga, G., et al., "Selective serotonin reuptakeinhibitors - a new modality for the treatment of lymphoma/ leukaemia?", Biochemical Pharmacology, no. 74(9), 2007

[13] Amit, B. H., Gil-Ad, I., Taler, M., Bar, M., Zolokov, A., Weizman, A., "Proapoptotic and chemosensiting effects of selective serotonin reuptake inhibitors on T cell lymphoma/ leukemia (Jurkat) in vitro", European Neuropsychipharmacology, no. 19(10), 2009

[14] A M David, S Nastase, CALITATEA VIEȚII, XXIII, nr. 1, 2012

[15] Dobson, K., Handbook of cognitive-behavioral therapies (2nd ed.), New York, The Guilford Press, 2001. 